\title{
Ovos produzidos por rainhas e operárias de Scaptotrigona depilis (Hymenoptera, Apidae, Meliponina): morfometria e aspectos relacionados
}

\author{
Lenira M.Lacerda ${ }^{1} \&$ Zilá L. P. Simões ${ }^{2}$
}

\begin{abstract}
1. Departamento de Biologia, Universidade Federal do Maranhão, Av. dos Portugueses, Campus do Bacanga, 65040-080 São Luís, MA, Brasil. (lenlacer@ufma.br)

2. Departamento de Biologia, Faculdade de Filosofia, Ciências e Letras de Ribeirão Preto, USP, Av. dos Bandeirantes, 3.900, Monte Alegre, 14040-901 Ribeirão Preto, SP, Brasil. (zlpsimoe@rge.fmrp.usp.br)
\end{abstract}

\begin{abstract}
Eggs produced by queens and workers of Scaptotrigona depilis (Hymenoptera, Apidae, Meliponina): morphometry and related aspects. The morphometric characterization of 785 eggs laid by queens and 193 (161 functional and 32 trophic eggs) laid by workers of Scaptotrigona depilis (Moure, 1942) has shown that they are similar in size and shape to the ones from other species of Meliponina. In the same colony, the queen's eggs always presented significant smaller size than the ones of the workers (functional or trophic). Among the workers' eggs, the functional eggs were, usually, shorter and narrower than the trophic eggs. The study of eggs laid by the queen has not shown, as expected, the presence of two groups of eggs with distinctive length, as observed in S. postica (Latreille, 1807).
\end{abstract}

KEYWORDS. Eggs, functional, trophic, males, Scaptotrigona.

RESUMO. A caracterização morfométrica de 785 ovos produzidos por rainhas e 193 (161 funcionais e 32 tróficos) produzidos por operárias de Scaptotrigona depilis (Moure, 1942), mostrou que eles são similares, em tamanho e formato, aos de outras espécies de abelhas Meliponina. Numa mesma colônia, ovos de rainha sempre apresentaram menor comprimento que os de operárias (funcionais ou tróficos). Entre ovos produzidos por operárias, ovos funcionais foram, normalmente, mais curtos e estreitos que os tróficos. O estudo dos ovos produzidos por rainhas não mostrou, como esperado, a presença de dois grupos de ovos distinguíveis pelo comprimento, como observado em S. postica (Latreille, 1807).

PALAVRAS-CHAVE. Ovos, funcional, trófico, machos, Scaptotrigona.

A reprodução das abelhas da subtribo Meliponina não é uma atribuição exclusiva da rainha. As operárias da maior parte das espécies também podem desenvolver ovários, realizar postura e produzir machos, mesmo na presença da rainha (SAKAGAMi et al., 1963; SAKAGAMI \& ZuCChi, 1966; SAKAgAmi, 1982; CruZ-LandiM, 2000; Toth, Queller et al., 2002; Toth, Strassman et al., 2002; PAXTON et al., 2003; Тотн et al., 2004).

Os ovos produzidos por operárias podem servir de alimento para rainha (algumas vezes para as operárias), ou originar machos. Por esta razão, vêm recebendo diferentes denominações como: alimentares, nutritivos, tróficos, funcionais e reprodutivos (CRUZ-LANDIM \& Höfling, 1971; Wilson, 1971; Bego, 1982; Sommeijer \& VAN Buren, 1992).

Diferentes parâmetros têm sido utilizados para a caracterização de ovos tróficos e funcionais. Dentre esses, destacam-se o formato, a coloração, e a presença ou ausência de retículo coriônico (SAKAGAMI \& ZuCCHI, 1963; SAKAGAMI, 1982; SommeiJer et al., 1984).

Da CunHa (1988) verificou que ovócitos maduros, nutritivos e funcionais, apresentam lipídeos e glicogênio. Os nutritivos, entretanto, diferenciam-se por apresentarem esferas protéicas imaturas e grande quantidade de núcleos acessórios.

Ovos tróficos de Scaptotrigona postica (Latreille, 1807), postos principalmente na margem das células (SAKAGAmi \& Zucchi, 1963), são citoquimicamente imaturos (CRUZ-LANDim \& HöFLING, 1971) e não apresentam membrana nuclear (AKAHIRA et al., 1970); por isto são considerados inviáveis. Os funcionais são depositados mais comumente no interior das células aprovisionadas onde a rainha já efetuou a postura e originam machos. Isto ocorre porque a larva de operária - proveniente do ovo posto pela rainha - é morta pela larva de macho originada do ovo da operária (BEIG, 1972). Resultados obtidos por L. M. Lacerda (dados não publicados) e KoEDAm et al. (2005) sugerem entretanto, que este princípio (larvofagia obrigatória) parece não ser um padrão para as espécies de Meliponina.

As rainhas de $S$. postica, por outro lado, também podem produzir machos (BEIG, 1972; РAXTON et al., 2003) a partir da postura de ovos (haplóides), estatisticamente maiores que aqueles responsáveis pela produção de operárias (diplóides) (BEIG, 1972). Esta constatação permitiu o surgimento de estudos que focalizam os mecanismos envolvidos na dinâmica da produção de machos por operárias e rainhas, resultante do conflito de interesses genéticos (RATNIEKS, 1988; Toth, STRASSMAN et al., 2002, Тотн et al., 2004; Velthuis et al., 2005) entre as castas.

Este trabalho teve como objetivo caracterizar os ovos produzidos por rainhas e operárias de Scaptotrigona depilis (Moure, 1942), procurando diferenciá-los por meio de análise morfométrica. Adicionalmente, pretendeu-se verificar, por meio do mesmo método, se os ovos 
produzidos pelas rainhas poderiam ser, à semelhança do que foi verificado em $S$. postica, separados em dois grupos de diferentes tamanhos.

\section{MATERIAL E MÉTODOS}

Os ovos, 785 de rainhas e 193 de operárias (161 funcionais e 32 tróficos) foram coletados entre dezembro de 1996 e abril de 1999 , em 10 colônias mantidas a $28^{\circ} \mathrm{C}$ (SAKAGAmi, 1966).

O comprimento $(\mathrm{C})$ e as larguras anterior (L1), mediana (L2) e posterior (L3), foram medidos em estereomicroscópio, com aumento de 40X.

As colônias das quais os ovos foram retirados apresentavam características típicas de colônias fortes (LACERDA et al., 1991): os favos apresentavam diâmetro de $\pm 10 \mathrm{~cm}$, os processos de oviposição (POPs Provisioning Oviposition Process, conforme ZuCCHI et al., 1999) ocorriam a cada \pm 180 min e envolviam a postura de 15 a 30 ovos pela rainha.

Todas as colônias receberam, a cada quatro dias, alimento ad libitum constituído de uma mistura de solução de água e açúcar (próximo à saturação) e pólen (de Apis, comercial, desidratado).

A despeito das várias denominações dadas aos ovos de operárias, foram designados funcionais aqueles ovos de forma alongada, e de tróficos aqueles de forma mais arredondada. Ovos de rainha e ovos funcionais foram retirados do interior da célula por meio de uma micropipeta, juntamente com o alimento semilíquido. Os ovos tróficos, normalmente postos na margem das células, foram retirados imediatamente após a sua postura por meio de um pequeno pincel embebido com mel para melhor adesão. Para determinar a procedência dos ovos retirados de células já operculadas, ovos de rainhas e operárias foram padronizados previamente, segundo seu comprimento.

Como os ovos retirados do interior das células apresentavam embriões com diferentes idades, foi necessário verificar se este aspecto poderia influenciar o tamanho dos ovos. Para isto, 53 ovos de rainha com idades entre 0 e 25 horas (divididos em 8 grupos) foram medidos. O limite máximo de 25 horas após a postura foi estabelecido porque neste período inicia-se o aparecimento de maior espaço entre o córion e a membrana vitelínica na porção posterior do ovo, o que pode ser facilmente observado sob a lupa.

Com o objetivo de aumentar a amostragem dos ovos funcionais (postos menos freqüentemente), testouse se ovos com mais de 25 horas diferiam de outros mais jovens. Em caso negativo, o limite de idade poderia ser estendido, facilitando assim a obtenção de maior amostragem em um menor espaço de tempo.

Para análise estatística foram usados os testes de Kruskall Wallis, t de Student e de Mann-Whitney (SoKAL \& RoHLF, 1995).

Operárias, rainhas e machos das colônias utilizadas neste trabalho estão depositados na Coleção Entomológica do Departamento de Biologia da Faculdade de Filosofia, Ciências e Letras de Ribeirão Preto (USP), sob a responsabilidade do Prof. Dr. João M. F. de Camargo.

\section{RESULTADOS}

O teste aplicado sobre o comprimento dos ovos de rainhas contendo embrião de diferentes idades não indicou variação significativa $(H=15,09, p>0,05, n=53)$. Da mesma forma, o comprimento dos ovos de operárias com idades de 0 a $25 \mathrm{~h}$ não diferiu significativamente daqueles com idade superior $(t=0,724, p>0,05, n=69)$. Por esta razão, os últimos foram incorporados à amostra, inicialmente constituída apenas por ovos com idade inferior a $25 \mathrm{~h}$.

Ovos postos pelas rainhas e ovos funcionais são alongados, ligeiramente curvos, e de aspecto hialino, mesmo sob luz refletida. A região anterior é ligeiramente mais larga que a posterior (Figs. 1, 2) e a região oposta não apresenta o córion reticulado característico do restante do ovo.

Ovos tróficos são mais arredondados (Fig. 3) e nem sempre é possível distinguir porção anterior e posterior dos mesmos. São amarelados e apresentam córion sem retículo coriônico evidente. Eles não têm a turgescência dos demais ovos, são extremamente frágeis, de difícil manuseio, e a distribuição do vitelo freqüentemente é desigual.
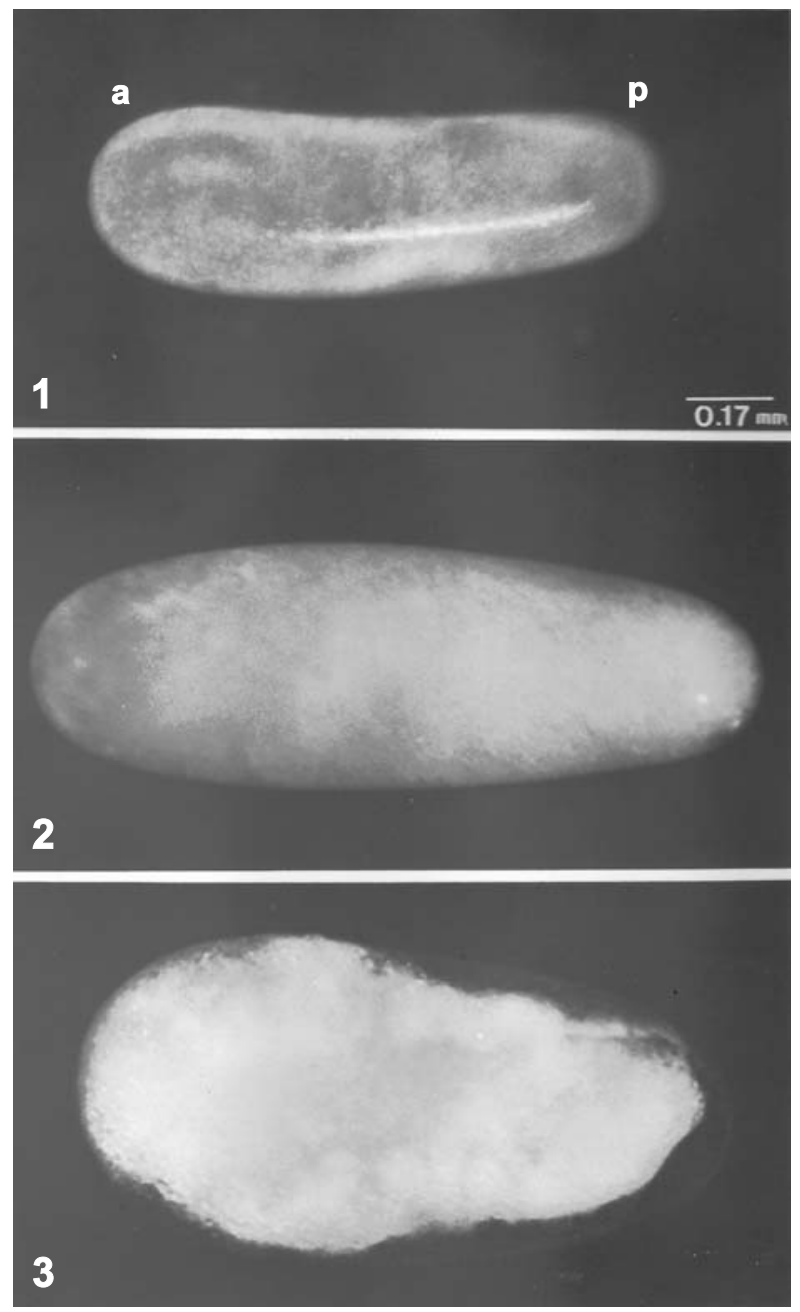

Figs. 1-3. Ovos postos por rainha (1) e operárias (2, funcional; 3, trófico) de Scaptotrigona depilis (Moure, 1942). A escala em 1 é válida para os três ovos (a, porção anterior; p, porção posterior). 
Tabela I. Caracterização morfométrica de ovos produzidos por rainhas e operárias de Scaptotrigona depilis (Moure, 1942) e S. postica (Latreille, 1807) (1, diferença significativa; 2, desvio padrão não-disponível; C, comprimento; L1, largura anterior; L2, largura mediana; L3, largura posterior).

\begin{tabular}{|c|c|c|c|c|c|}
\hline Espécie & Casta & $\mathrm{C}(\mathrm{mm})$ & $\mathrm{L} 1$ (mm) & $\mathrm{L} 2(\mathrm{~mm})$ & L3 (mm) \\
\hline \multirow{3}{*}{ S. depilis } & rainha & $\begin{array}{c}1,16 \pm 0,721^{1} \\
(\mathrm{n}=785)\end{array}$ & $\begin{array}{c}0,40 \pm 0,030^{1} \\
(n=413)\end{array}$ & $\begin{array}{c}0,39 \pm 0,028^{1} \\
(n=413)\end{array}$ & $\begin{array}{l}0,34 \pm 0,028^{1} \\
\quad(\mathrm{n}=412)\end{array}$ \\
\hline & funcional & $\begin{array}{c}1,32 \pm 0,060^{1} \\
(\mathrm{n}=169)\end{array}$ & $\begin{array}{c}0,41 \pm 0,030^{1} \\
(n=104)\end{array}$ & $\begin{array}{c}0,40 \pm 0,024^{1} \\
(n=108)\end{array}$ & $\begin{array}{l}0,35 \pm 0,046^{1} \\
(\mathrm{n}=103)\end{array}$ \\
\hline & trófico & $\begin{array}{c}1,54 \pm 0,126 \\
(\mathrm{n}=33)\end{array}$ & $\begin{array}{c}0,54 \pm 0,120 \\
(n=9)\end{array}$ & $\begin{array}{c}0,67 \pm 0,087 \\
(n=29)\end{array}$ & $\begin{array}{c}0,54 \pm 0,1116 \\
(\mathrm{n}=18)\end{array}$ \\
\hline $\begin{array}{c}\text { S. postica } \\
\text { (AKAHIRA et al., 1970) }\end{array}$ & $\begin{array}{c}\text { rainha } \\
\text { operária/trófico }\end{array}$ & $\begin{array}{l}1,20^{2} \\
1,50^{2}\end{array}$ & - & $\begin{array}{l}0,30^{2} \\
0,77^{2}\end{array}$ & - \\
\hline $\begin{array}{l}\text { S. postica } \\
\text { (CRuZ-LANDim \& HöFling, 1971) }\end{array}$ & $\begin{array}{l}\text { rainha } \\
\text { operária/trófico }\end{array}$ & $\begin{array}{l}0,828^{2} \\
0,869^{2}\end{array}$ & - & $\begin{array}{l}0,273^{2} \\
0,434^{2}\end{array}$ & - \\
\hline $\begin{array}{l}\text { S. postica } \\
(\text { BEIG, 1972) }\end{array}$ & $\begin{array}{l}\text { rainha/diplóide } \\
\text { rainha/haplóide } \\
\text { operária/funcional }\end{array}$ & $\begin{array}{l}1,185 \pm 0,018 \\
1,315 \pm 0,033 \\
1,305 \pm 0,033\end{array}$ & - & - & - \\
\hline
\end{tabular}

Excluídos os ovos tróficos, claramente diferentes dos demais, os testes aplicados nas medidas de ovos da rainha e ovos funcionais indicaram diferenças significativas em todas as medidas $(\mathrm{C}: \mathrm{z}=19,34, \mathrm{p}<0,01$; $\mathrm{L} 1: \mathrm{z}=4,19, \mathrm{p}<0,01 ; \mathrm{L} 2: \mathrm{z}=8 ; 43, \mathrm{p}<0,01 ; \mathrm{L} 3: \mathrm{z}=4 ; 13$, $\mathrm{p}<0,01)$ (Tab. I).
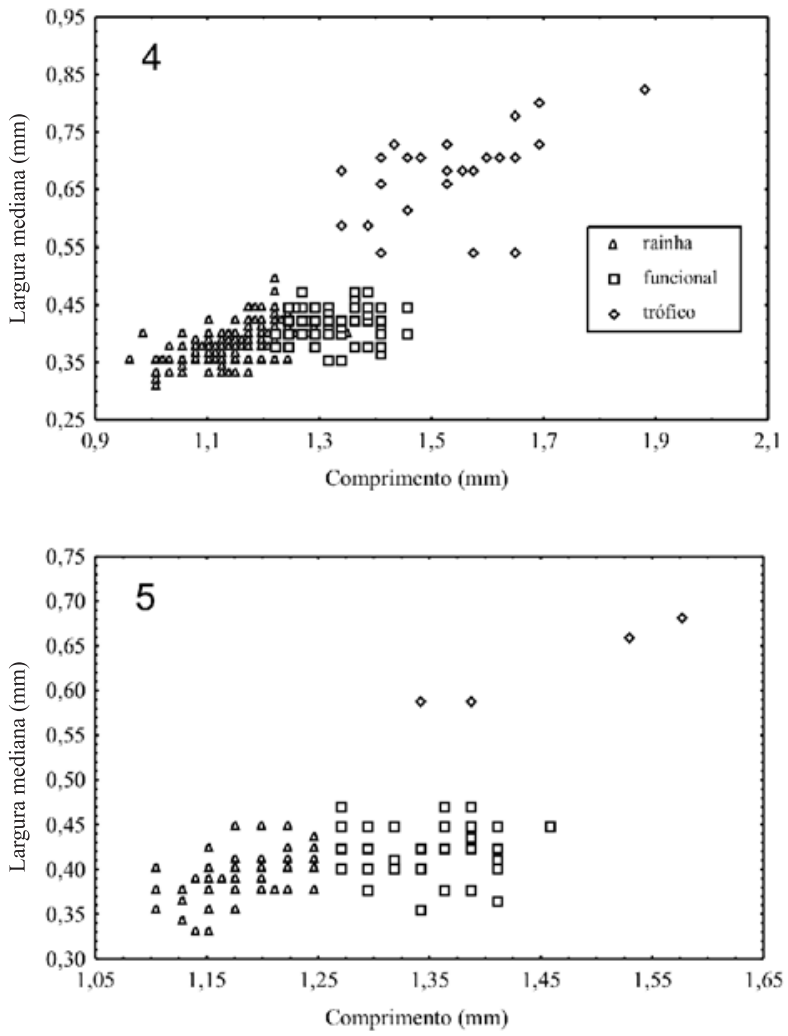

Figs. 4, 5. Análise morfométrica de ovos depositados por fêmeas de Scaptotrigona depilis (Moure, 1942): 4, ovos obtidos em 10 colônias (785 ovos de rainhas, 161 funcionais e 32 tróficos); 5 , ovos obtidos em apenas uma colônia ( $\left.\mathrm{n}^{\circ} 4\right)$ (121 ovos da rainha, 68 funcionais e 4 tróficos).
O comprimento e a largura mediana foram as medidas que melhor separaram os três tipos de ovos $(\mathrm{C}$ : $\mathrm{H}=445,23$; $\mathrm{L} 2: \mathrm{H}=150,48$; em ambos, $\mathrm{p}<0,01)$, sobretudo os tróficos (Figs. 1-3, Tab. I).

Observou-se uma faixa de sobreposição do comprimento dos ovos da rainha e ovos funcionais (Fig. 4), sugerindo que as duas castas poderiam produzir, numa mesma colônia, ovos de igual tamanho. Não obstante, raros foram os casos de sobreposição de comprimento, quando a análise foi feita em cada colônia separadamente, como por exemplo na colônia $n^{\circ} 4$ (Fig. 5).

Quanto aos ovos depositados pelas rainhas, a distribuição dos valores do comprimento - todas as colônias juntas, ou separadamente - não mostrou qualquer descontinuidade capaz de separar ovos maiores e menores.

\section{DISCUSSÃO}

Influência da idade do embrião sobre o comprimento dos ovos. Diferentemente dos resultados obtidos por Woyke (1992) em três espécies de Apis, a idade do embrião de $S$. depilis não influenciou o comprimento dos ovos. A ausência de redução de tamanho ao longo do tempo, como verificado em Apis, pode ser atribuída a dois fatores: 1 , os ovos de $S$. depilis são postos sobre o alimento semilíquido e a célula é totalmente fechada segundos após a postura; desta forma, a chance de desidratação dos mesmos é menor que a dos ovos de Apis, que são depositados no fundo de células vazias permanentemente abertas; 2, o período de observação dos ovos neste trabalho foi mais reduzido ( 0 a 25 horas) que o de Apis (0 a 52 horas), diminuindo ainda mais a possibilidades de desidratação.

Morfometria dos ovos de rainhas e operárias. Os ovos de $S$. depilis mostraram-se semelhantes aos de outras espécies de Meliponina, como Melipona quadrifasciata anthidioides Lepeletier, 1836 (CRUZLANDIM, 1966; CRUZ-LANDIM \& YABUKI, 1995); S. postica (Akahira et al., 1970; BeIG, 1971, 1972; CruZ-Landim \& 
Höfling, 1971 (Tab. I); CRUZ-LANDIM \& YABUKI, 1995); Melipona rufiventris paraensis Ducke, 1916 (SOMMEIJER et al., 1984); Trigona (Tetragonisca) angustula Illiger, 1811 (Koedam et al., 1996); Apis mellifera Linnaeus, 1758 (CRUZ-LANDIM \& YABUKI, 1995).

O comprimento médio dos ovos das rainhas de $S$. depilis $(1,16 \mathrm{~mm})$ foi muito próximo ao valor encontrado por BeIG (1972) para ovos diplóides de $S$. postica $(1,18$ mm) (Tab. I). Ovos com comprimento idêntico ao comprimento médio de ovos haplóides da rainha de $S$. postica constituíram apenas $7,0 \%$ do total de ovos depositados, e foram obtidos em somente 3 das 10 colônias estudadas. Este dado sugere que, se ovos com tal dimensão forem realmente haplóides (como em $S$. postica), apenas um terço das rainhas de $S$. depilis (colônias fortes) utilizadas neste trabalho teriam produzido machos no prazo de três anos. Entretanto, há registros (PAXTON et al., 2003) e indícios (L. M. Lacerda, dados não publicados) de que rainhas desta espécie podem produzir até $100 \%$ dos machos da colônia. Assim, uma suposta relação entre tamanho do ovo e o sexo do embrião em $S$. depilis parece não ser simples nem direta e ainda não está claramente esclarecida.

No período de coleta dos ovos de maior tamanho (janeiro, abril, maio, agosto, setembro e dezembro), a porcentagem de células contendo mais que um ovo variou entre 0 e $50 \%$. Desta forma, supõe-se que, caso estes ovos de rainha fossem haplóides, a rainha poderia produzi-los sozinha ou simultaneamente com as operárias. Todavia, a despeito de algumas tentativas de acompanhamento do desenvolvimento dos ovos medidos para saber o sexo do embrião, não foi possível qualquer comprovação da haploidia destes.

Como a produção de machos em S. postica, independente da origem dos mesmos, pode ocorrer em diferentes épocas do ano (BEIG, 1972; BEGO, 1982; ENGELS \& ENGELs, 1984; PAXton et al., 2003), não há como testar uma correlação entre época de produção de ovos de maior tamanho e a época de produção de machos.

A nomenclatura dos ovos postos por operárias vem, ao longo dos últimos anos, tornando-se cada vez mais genérica, afastando-se progressivamente das caracterizações formuladas originalmente. Tais generalizações têm-se originado não só do crescente número de espécies que vêm sendo estudadas, como também da grande variabilidade de comportamento apresentados pelas operárias de diferentes espécies. Além disto, algumas características como formato, coloração, local da postura, presença de retículo coriônico e viabilidade dos ovos (parâmetros amplamente utilizados para melhor caracterização dos mesmos), freqüentemente se sobrepõem e não são mutuamente exclusivas.

Parâmetro como local de postura e função dos ovos tem gerado algumas incongruências, como por exemplo: ovos de forma alongada, chamados originalmente de funcionais e, mais tarde, de reprodutivos, ao contrário do esperado, não se desenvolvem quando são depositados sobre o favo ou às margens das células e ingeridos pela rainha ou pelas operárias (SAKAGAMI \& Zucchi, 1963, 1967; WitTMAnn et al., 1991; Drumond, Zucchi, Yamane \& Sakagami, 1996; Drumond, Zucchi, Mateus et al., 1996; LaCerda et al., 1998; Nogueira-
FERREIRA et al., 1998). Esse mesmo tipo de ovo, em outras espécies, ainda que posto no interior das células aprovisionadas, também não se desenvolve, porque é freqüentemente ingerido pela rainha (SAKAGAMI et al., 1965). Assim, um mesmo ovo pode ser, simultaneamente, considerado funcional, pelo seu formato, e trófico, por ter servido de alimento para a rainha.

Embora a funcionalidade do ovo possa ser uma característica válida para caracterização dos mesmos, esta requer tempo e condições especiais para ser confirmada. Até o momento, apenas três espécies foram, sistematicamente, estudadas quanto ao desenvolvimento dos ovos até a emersão dos adultos: S. postica (BEIG, 1972), Melipona subnitida Ducke, 1910 (Koedam et al., 1999) e Melipona favosa Fabricius, 1789 (SOMMEIJER \& VAN BuREN, 1992).

Diante dessas considerações e do pouco conhecimento acerca dos dois tipos de ovos, sugere-se que o melhor parâmetro para definição dos mesmos ainda seja seu formato: alongado ou arredondado. Este caráter, além de ser facilmente visível a olho nu, não tem, nas espécies estudadas, apresentado significativas variações exceção feita a espécies de Plebeia, nas quais, nem sempre, há distinção entre os dois tipos (DRUMOND, ZuCChI, YAMANE \& SAKAGAMI, 1996). Os outros atributos relativos ao local no qual foi depositado, localização temporal dentro do POP, e autoria da oofagia, seriam informações mais secundárias neste aspecto, porém não menos importantes para o entendimento do papel destes ovos num contexto social mais amplo.

Agradecimentos. Este trabalho constituiu parte da tese de doutoramento financiado pela CAPES, CNPq e FAPEMA. Agradecemos especialmente ao Prof. Dr. Ronaldo Zucchi, FFCLRP - USP, e Profa. Gisele G. Azevedo, UFMA, pelas valiosas sugestões. Ao Dr. Sidnei Mateus, pela assistência na manutenção das colônias.

\section{REFERÊNCIAS BIBLIOGRÁFICAS}

Akahira, Y.; Sakagami, S. F. \& Zucchi, R. 1970. Die Nähreier von den Arbeiterinnen einer stachellosen Bien, Trigona (Scaptotrigona) postica, die von Königin Kurz vor der eigenen Eiablage gefressen werden. Sonderdruck Zoologgischer Anzeiger 185(1/2):85-93.

Bego, L. R. 1982. On social regulation in Nannotrigona (Scaptotrigona) postica Latreille, with special reference to male production cycles (Hymenoptera, Apidae, Meliponinae). Boletim de Zoologia da Universidade de São Paulo 7:181-196.

BeIG, D. 1971. Desenvolvimento embrionário de abelhas operárias de Trigona (Scaptotrigona) postica Latreille (Hymenoptera, Meliponinae). Arquivos de Zoologia 21(4):179-234.

1972. The production of males in queenright colonies of Trigona (Scaptotrigona) postica. Journal Apiculture Research 11(1):33-39.

Cruz-Landim, C. 1966. Alguns dados sobre o desenvolvimento de "Melipona" (Hym. Apoidea). Revista Brasileira de Biologia 26(2): 165-174.

2000. Ovarian development in Meliponinae bees (Hymenoptera, Apidae): the effect of queen presence and food on worker ovary development and egg production. Genetics and Molecular Biology 23:83-88

Cruz-Landim, C. \& Höfling, M. A. DA. 1971. Cytochemical and ultrastrutuctural studies on eggs from workers and queen of Trigona. Revista Brasileira Pesquisas Médicas e Biológicas 4(1-2):19-25.

Cruz-Landim, C. \& Yabuki, A. T. 1995. Fine structure and morphogenesis of the micropyle apparatus in bees eggs. 
Biocell 19(2):125-132.

Da Cunha, M. A. S. 1988. Ultrastructure of the oocytes of Scaptotrigona posiuca workers (Hymenoprtera, Apidae). Naturalia 13:103-115.

Drumond, P.; Zucchi, R.; Yamane, S. \& SaKagami, F. S. 1996. Oviposition behaviour of the stingless bees XVIII. Plebeia (Plebeia) emerina and $P$. $(P$.) remota with a preliminaray ethological comparison of some Plebeia taxa (Apidae, Meliponinae). Natural History Bulletim Ibaraki University 45:31-55.

Drumond, P.; Zucchi, R.; Mateus, S.; Bego, L. R. \& Sakagami, F. S. 1996. Oviposition behavior of the stingless bees XVII. Plebeia (Plebeia) droryana and an ethological comparison with other Meliponinae taxa (Hymenoptera, Apidae). Japonese Journal of Entomology 64(2):385-400.

EngELS, E. \& ENGELs, W. 1984. Drohnen-ansammungen bei nestern der stachellosen biene Scaptotrigona postica. Apidologie 15(3):315-328.

Koedam, D.; Contrera, F. A. L. \& Imperatriz-Fonseca, V. L. 1999. Clustered male production by workers in the stingless bees Melipona subnitida Ducke (Apidae, Meliponinae). Insectes Sociaux 46:387-391.

2005. How queen workers share in male production in the stingless bee Melipona subnitida Ducke (Apidae, Meliponini). Insectes Sociaux 52:114-121.

Koedam, D.; Velthuiz, P. H.; Krift, T. V. D.; Dohmen, M. R. \& SommeiJer, M. J. 1996. Morphology of reproductive and trophic eggs and their controlled release by workers in Trigona (Tetragonisca) angustula Illiger (Apidae, Meliponinae). Physiological Entomology 21:289-296.

LACERDA, L. DE M.; ZuCChI; R. \& SAKAGAMI, F. S. 1998. Oviposition behavior of the stingless bees, XXV. Ethological relationship of Geotrigona mombuca to other stingless bees taxa (Insecta: Hymenoptera; Apidae, Meliponinae). Natural History Bulletim of Ibaraki University, 2:263-276.

Lacerda, L. De M.; Zucchi, R. \& Zucoloto, F. S. 1991. Colony condition and bionomic alterations em Geotrigona inusitata (Apidae, Meliponinae) Acta Biologica Paranaense 20(1/ 4):109-123.

Nogueira-Ferreira, F. H.; Zucchi, R.; Silva-Matos, E. V. Da \& SaKagami, S. F. 1998. Oviposition behavior of stingless bees XXIII. Schwarziana quadripunctata with comments on ethological diversity among representatives of the Plebeia complex (Hymenoptera, Apidae, Meliponinae). Entomological Science 1(4):511-521.

Paxton, R. J.; Bego, L. R. \& Shah, M. M. 2003. Low mating frequency of queens in the stingless bee Scaptotrigona postica and worker maternity of males. Behavioral Ecology and Sociobiology 53:174-181.

RATNIEKS, F. L. W. 1988. Reprodutive harmony via mutual policing by workers in eusocial hymenoptera. American Naturalist 132:217-236

SAKagami, S. F. 1966. Techniques for the observation of behaviour and social organization of stingless bees by using a special hive. Papéis Avulsos do Departamento de Zoologia 19:151-162.

1982. Stingless bees. In: Hermann, R. H. ed. Social insects. New York, Academic Press. v.3, p.316-423.

Sakagami, S. F. \& Zucchi, R. 1963. Oviposition process in a stingless bee Trigona (Scaptotrigona) postica Letr. (Hymenoptera). Studia Entomologica 6(1/4):497-510. 1966. Estudo comparativo do comportamento de várias espécies de abelhas sem ferrão, com especial referência ao processo de aprovisionamento e postura das células. Ciência e Cultura 18:283-296.

1967. Behavior studies of the stingless bees, with special reference to the oviposition process. VI. Trigona (Tetragona) clavipes. Journal of the Faculty of Science, Hokkaido University 16(2):292-313

Sakagami, S. F; Montenegro, M. J. \& Kerr, W. E. 1965. Behavior studies of the stingless bees, with special reference to the oviposition process. V. Melipona quadrifasciata anthidioides Lepeletier. Journal of the Faculty of Science, Hokkaido University 15(4):578-607.

Sakagami, S. F.; Beig, D.; Zucchi, R. \& Akahira, Y. 1963. Occurence of ovary-developed workers in queenright colonies of stingless bees. Revista Brasileira de Biologia 23(2):115-129.

SoKal, R. R. \& Rohlf, F. J. 1995. Biometry: the principles and practice of statistics in biological research. $3^{\text {rd }}$ ed. New York, W. H. Freeman. 887p.

Sommeijer, M. J. \& VAn Buren, N. W. M. 1992. Male production by laying workers in queenright colonies of Melipona favosa (Apidae, Meliponinae). In: Billen, J. ed. Biology and evolution of social Insects. Leuven, University Press. p.8997.

Sommeijer, M. J.; Van Zeijl, M. \& Dohmen, M. R. 1984. Morphological differences between worker-laid eggs from a queenright colony and queenless colony of Melipona rufiventris paraensis (Hymenoptera: Apidae). Entomologische Berichichten 44(1):91-95.

Toth, E.; Queller, D. C.; Dollin, A. \& Strassmann, J. E. 2004. Conflict over male parentage in stingless bees. Insecte Sociaux 51:1-11.

Toth, E.; Queller, D. C.; Imperatriz-Fonseca, V. L. \& Strassmann, J. E. 2002. Genetic and behavioral conflict over male paroduction between workers and queens in the stingless bee Paratrigona subnuda. Behavioral Ecology and Sociobiology 53:1-8.

Toth, E.; Strassmann, J. E.; Nogueira-Neto, P.; Imperatriz-Fonseca, V. L. \& Queller, D. 2002. Male production in stingless bees: variable outcomes of queen-worker conflict. Molecular Ecology 11:2661-2667.

Velthuis, H. H. W.; Koedam, D. \& Imperatriz-Fonseca, V. L. 2005. Males of Melipona and other stingless bees, and their mothers. Apidologie 36:169-185.

Wilson, E. O. 1971. The insect societies. Cambridge, Belknap. $548 \mathrm{p}$.

Wittmann, D.; Bego, L. R.; Zucchi, R. \& Sakagami, S. F. 1991. Oviposition behavior and related aspects of the stingless bees XIV. Plebeia (Plebeia) caerulea, with comparative notes on the evolution of the oviposition patterns (Apidae, Meliponinae). Japanese Journal of Entomology 59(4):793-809.

Woyke, J. 1992. Size change of eggs during the incubation period um three Asian honey bee species. In: Connor, L. J.; Rinderer, T; Silvester, A. \& Wongsiri, S. eds. Asian apiculture: proceedings of first international conference on asian honey bees and bee mites. Cheshire, Wicwas Press p.197205 .

Zucchi, R.; Silva-Matos, E. V. da; Nogueira-Ferreira, F. H. \& Azevedo, G. G. 1999. On the cell provisioning and oviposition process (POP) of the stingless bees nomenclature reappraisal and evolutionary considerations (Hymenoptera, Apidae, Meliponinae). Sociobiology 34(1):65-86.

Recebido em junho de 2005. Aceito em dezembro de 2005. ISSN 0073-4721

Artigo disponível em: www.scielo.br/isz 